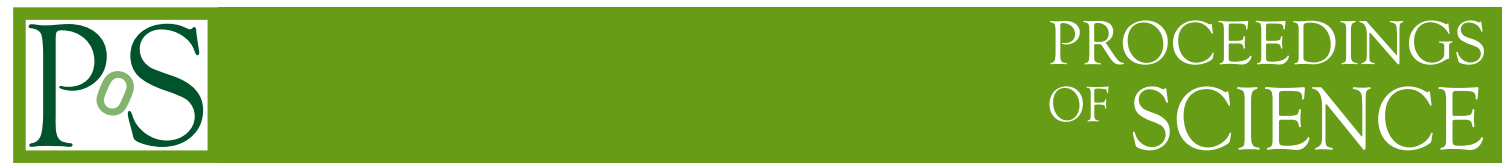

\title{
Charmonia production in heavy-ion collisions: status and perspectives
}

\section{E. Scomparin*}

INFN Torino, Via P. Giuria 1, I-10125 Torino (Italy)

E-mail: scomparin@to.infn.it

\begin{abstract}
The study of charmonium production in nuclear collisions has started in the 80's, when the suppression of bound $c \bar{c}$ states was predicted as a signature of the transition from an hadronic system towards a Quark-Gluon Plasma. At the SPS, and then at RHIC, systematic studies of this observable have been carried out, in both nucleus-nucleus and proton-nucleus collisions. With the advent of the LHC new results in an unexplored energy range are about to come. In this paper, we report on recent results on this topic and on the perspectives for the near future.
\end{abstract}

XLVIII International Winter Meeting on Nuclear Physics, BORMIO2010

January 25-29, 2010

Bormio, Italy

${ }^{*}$ Speaker. 


\section{Introduction}

Heavy quarkonia states represent an interesting testing groud of our comprehension of QCD. The production of the $q \bar{q}$ pair and its bounding involve perturbative and non-perturbative aspects of the theory, respectively. Today, more than 30 years after the discovery of the $J / \psi$ state, significant progress has been made in the understanding of charmonium hadroproduction, even if a coherent description of all the experimental observables ( $p_{\mathrm{T}}$ distributions, polarization) is still not available [1]. In spite of this limitation, heavy quarkonium (and, in particular, charmonium) has also been used a probe of the hot medium created in nuclear collisions. In particular, if a deconfined system of quarks and gluons is created, colour screening should prevent the formation of a bound $c \bar{c}$ state [2]. However, several effects may act together to produce a suppression of the charmonium states. In particular, also in $p A$ collisions a sizeable charmonium suppression has been observed. This suppression is due to various initial and final state effects in cold nuclear matter and its existence has to be correctly taken into account when trying to isolate a genuine signal of the so-called "anomalous" suppression, due to hot matter effects, in $A A$ collisions. Today, thanks to systematic studies mainly carried out by the NA50 and NA60 experiments at the CERN SPS, and by the PHENIX experiment at RHIC, the anomalous $\mathrm{J} / \psi$ suppression has been investigated in great detail. In the following sections, I report on recent observations carried out at SPS and RHIC energies. Finally, I discuss the expectations for the study of charmonia and bottomonia suppression at the LHC.

\section{Recent results at SPS energy}

In recent years, studies of charmonium production and suppression in cold and hot nuclear matter have been carried out by the NA60 collaboration $[3,4,5]$. In particular, data have been taken for In+In collisions at $158 \mathrm{GeV} /$ nucleon and for $p A$ collisions at 158 and $400 \mathrm{GeV}$. In the following, the primary NA60 results and their impact on the understanding of the anomalous $J / \psi$ suppression, first observed by the NA50 collaboration in $\mathrm{Pb}+\mathrm{Pb}$ collisions [6], are summarized.

\section{1 $J / \psi$ production in $p A$ collisions at 158 and $400 \mathrm{GeV}$}

One of the main results of the SPS heavy-ion program was the observation of anomalous $J / \psi$ suppression. Results obtained in $\mathrm{Pb}+\mathrm{Pb}$ collisions at $158 \mathrm{GeV} /$ nucleon by the NA50 collaboration showed that, in such collisions, the $J / \psi$ yield was suppressed with respect to estimates that include only cold nuclear matter effects [6]. The magnitude of the cold nuclear matter effects has typically been extracted by extrapolating the $J / \psi$ production data obtained in $p A$ collisions. Until recently the reference SPS $p A$ data were based on samples collected at $400 / 450 \mathrm{GeV}$ by the NA50 collaboration, at higher energy than the nuclear collisions and in a slightly different rapidity domain $[7,8,9]$.

The need for reference $p A$ data taken under the same conditions as the $A A$ data was a primary motivation for the NA60 experiment. A run with an SPS primary proton beam at $158 \mathrm{GeV}$ was carried out in 2004. Seven nuclear targets $(\mathrm{Be}, \mathrm{Al} \mathrm{Cu}, \mathrm{In}, \mathrm{W}, \mathrm{Pb}$, and $\mathrm{U}$ ) were simultaneously exposed to the beam. During the same period, a $400 \mathrm{GeV} p A$ data sample was taken with the same experimental set-up. 


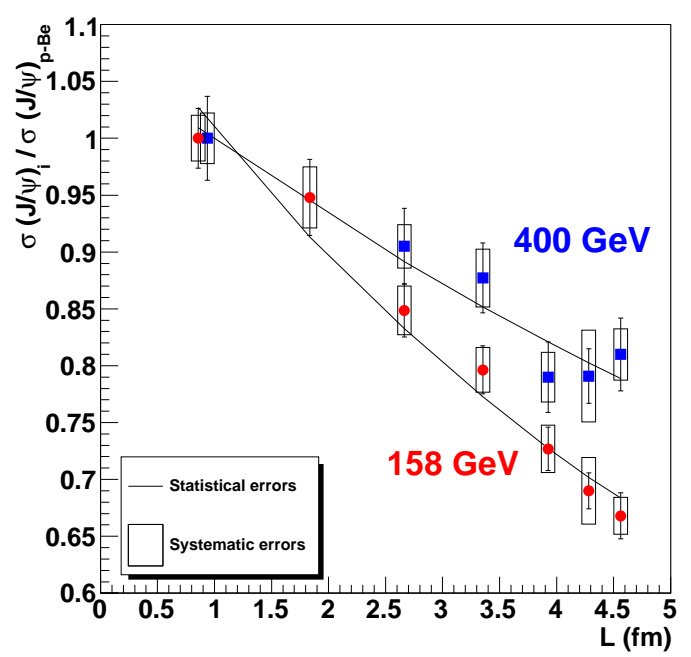

Figure 1: The $J / \psi$ cross section ratios for $p A$ collisions at $158 \mathrm{GeV}$ (circles) and $400 \mathrm{GeV}$ (squares), as a function of $L$, the mean thickness of nuclear matter traversed by the $J / \psi$.

Cold nuclear matter effects have been evaluated comparing the cross section ratio $\sigma_{p A}^{J / \psi} / \sigma_{p \mathrm{Be}}^{J / \psi}$, for each nucleus with mass number $A$, relative to the lightest target $(\mathrm{Be})$. The results [3,5], shown in Fig. 1, are integrated over $p_{T}$ and are given in the rapidity region covered by all the sub-targets, $0.28<y_{\mathrm{CMS}}<0.78$ for the $158 \mathrm{GeV}$ sample and $-0.17<y_{\mathrm{CMS}}<0.33$ for the $400 \mathrm{GeV}$ sample. Systematic errors include uncertainties in the target thickness, the rapidity distribution used in the acceptance calculation, and the reconstruction efficiency. Only the fraction of systematic errors not common to all the points is shown since it affects the evaluation of nuclear effects.

Nuclear effects have usually been parameterized by fitting the $A$ dependence of the $J / \psi$ production cross section using the expression $\sigma_{p A}^{J / \psi}=\sigma_{p p}^{J / \psi} A^{\alpha}$. Alternatively, the effective absorption cross section, $\sigma_{\mathrm{abs}}^{J / \psi}$ can be extracted from the data using the Glauber model. Both $\alpha$ and $\sigma_{\mathrm{abs}}^{J / \psi}$ are effective quantities since they represent the strength of the cold nuclear matter effects that reduce the $J / \psi$ yield. However, they cannot distinguish between the different effects, e.g. shadowing and nuclear absorption, contributing to this reduction. The results in Fig. 1 were used to extract $\sigma_{\text {abs }}^{J / \psi}=7.6 \pm 0.7$ (stat.) \pm 0.6 (syst.) $\mathrm{mb}$ (corresponding to $\alpha=0.882 \pm 0.009 \pm 0.008$ ) at $158 \mathrm{GeV}$ and $\sigma_{\text {abs }}^{J / \psi}=4.3 \pm 0.8$ (stat.) \pm 0.6 (syst.) $\left.\mathrm{mb}(\alpha=0.927 \pm 0.013 \pm 0.009)\right)$ at $400 \mathrm{GeV}$. Thus $\sigma_{\text {abs }}^{J / \psi}$ is larger at $158 \mathrm{GeV}$ than at $400 \mathrm{GeV}$. The $400 \mathrm{GeV}$ result is, on the other hand, in excellent agreement with the previous NA50 result obtained at the same energy [8].

The study of cold nuclear matter effects at fixed-target energies is a subject which has attracted considerable interest. In Fig. 2, a compilation of previous results for $\sigma_{\text {abs }}^{J / \psi}$ as a function of $x_{F}$ [7, $10,11,12$ ] is presented, together with the new NA60 results [5]. The values of $\sigma_{\mathrm{abs}}^{J / \psi}$ in Fig. 2 do not include any shadowing contribution, only absorption. There is a systematic increase in the nuclear effects going from low to high $x_{F}$ as well as when from high to low incident proton energies. As shown in Fig. 2, the new NA60 results at $400 \mathrm{GeV}$ confirm the NA50 values obtained at a similar energy. On the other hand, the NA60 $158 \mathrm{GeV}$ data suggest higher values of $\sigma_{\mathrm{abs}}^{J / \psi}$ as well a hint of increased absorption over the $x_{F}$ range. Note also that the older NA3 $J / \psi$ results are in 


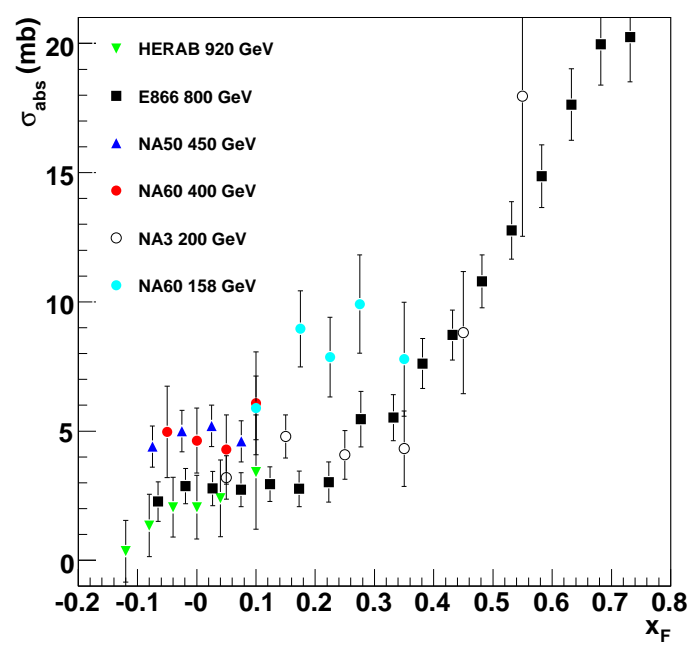

Figure 2: Compilation of $\sigma_{\text {abs }}^{J / \psi}$ as a function of $x_{F}$ with no additional cold matter effects included.

partial contradiction with these observations, giving lower values of $\sigma_{\mathrm{abs}}^{J / \psi}$, similar to those obtained from the higher energy data samples. Such a complex pattern of nuclear effects results from a delicate interplay of various nuclear effects (final state absorption, shadowing, initial state energy loss etc.) and has so far not been satisfactorily explained by theoretical models [13]. A first attempt at disentangling the contribution of shadowing from the effective absorption cross section $\sigma_{\mathrm{abs}}^{J / \psi}$ extracted from the NA60 results has been carried out, correcting the measured cross section ratios for the shadowing factors calculated using the EKS98 [14] parameterization of the nuclear PDFs. It was found that a larger $\sigma_{\mathrm{abs}}^{J / \psi}$ is needed to describe the measured data, $\sigma_{\mathrm{abs}}^{J / \psi}(158 \mathrm{GeV})=9.3 \pm 0.7 \pm$ $0.7 \mathrm{mb}$ and $\sigma_{\mathrm{abs}}^{J / \psi}(400 \mathrm{GeV})=6.0 \pm 0.9 \pm 0.7 \mathrm{mb}$. The results depend on the parameterization of the nuclear modifications of the PDFs. For example, slightly higher values of $\sigma_{\mathrm{abs}}^{J / \psi}$ (on the $\sim 5-10 \%$ level) are obtained if the EPS08 [15] parameterization is used.

\subsection{Anomalous $J / \psi$ suppression in $\mathbf{I n}+\mathbf{I n}$ and $\mathbf{P b}+\mathbf{P b}$ collisions}

The $p A$ results at $158 \mathrm{GeV}$ shown in the previous section have been collected at the same energy and in the same $x_{F}$ range of the SPS $A A$ data. It is therefore natural to use these results to calculate the expected magnitude of cold nuclear matter effects on $J / \psi$ production in nuclear collisions. In order to do so, the expected shape of the $J / \psi$ distribution as a function of the forward energy $E_{\mathrm{ZDC}}, d N_{\mathrm{J} / \psi}^{\text {expect }} / d E_{\mathrm{ZDC}}$, has been determined using the Glauber model. The $J / \psi$ yield is assumed to scale with the number of $N N$ collisions. The effective $J / \psi$ absorption cross section in nuclear matter is assumed to be the same as the value at $158 \mathrm{GeV}$ deduced in the previous section.

The measured $J / \psi$ yield, $d N_{\mathrm{J} / \psi} / d E_{\mathrm{ZDC}}$, is normalized to $d N_{\mathrm{J} / \psi}^{\text {expect }} / d E_{\mathrm{ZDC}}$ using the procedure detailed in Ref. [4]. This procedure previously did not take shadowing effects into account when extrapolating from $p A$ to $A A$ interactions. In $p A$ collisions, only the target partons are affected by shadowing while in $A A$ collisions, effects on both the projectile and target must be taken into account. If shadowing is neglected in the $p A$ to $A A$ extrapolation, a small bias is introduced, resulting in an artificial $\sim 5 \%$ suppression of the $J / \psi$ yield with the EKS98 parameterization [16]. 


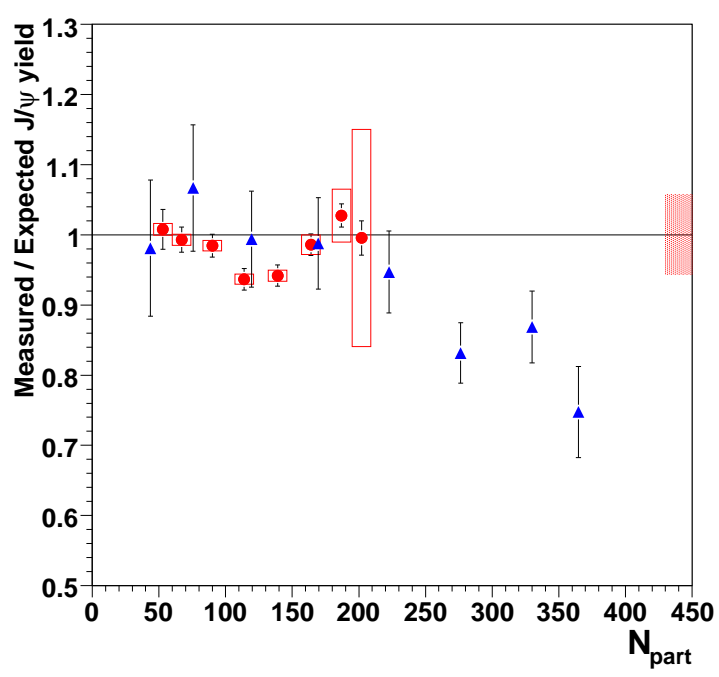

Figure 3: Anomalous $J / \psi$ suppression in $\mathrm{In}+\mathrm{In}$ (circles) and $\mathrm{Pb}+\mathrm{Pb}$ collisions (triangles), as a function of $N_{\text {part }}$. The boxes around the In+In points represent correlated systematic errors. The filled box on the righthand side corresponds to the uncertainty in the absolute normalization of the In+In points. A $12 \%$ global error, due to the uncertainty on $\sigma_{\text {abs }}^{J / \psi}$ at $158 \mathrm{GeV}$ is not shown.

Therefore, if shadowing is properly accounted for in the $p A$ to $A A$ extrapolation, the amount of the anomalous $J / \psi$ suppression is reduced. Figure 3 presents the results for the anomalous $J / \psi$ suppression in $\mathrm{In}+\mathrm{In}$ and $\mathrm{Pb}+\mathrm{Pb}$ collisions [3,5] as a function of $N_{\text {part }}$, the number of participant nucleons. Up to $N_{\text {part }} \sim 200$ the $J / \psi$ yield is, within errors, compatible with the extrapolation of cold nuclear matter effects. When $N_{\text {part }}>200$, there is an anomalous suppression of up to $\sim 20-30 \%$ in the most central $\mathrm{Pb}+\mathrm{Pb}$ collisions.

\section{Recent results at RHIC energy}

The last few years of the RHIC program have produced $J / \psi$ data from PHENIX for $\mathrm{d}+\mathrm{Au}$ and $\mathrm{Au}+\mathrm{Au}$ collisions with sufficient statistical precision to establish the centrality dependence of both hot and cold nuclear matter effects at $\sqrt{s_{N N}}=200 \mathrm{GeV}$. The data cover the rapidity range $|y|<2$.4. Both PHENIX and STAR have also studied $p p$ collisions [17, 18], extremely useful for a better understanding of nuclear collision results, and to test theoretical models of quarkonium hadroproduction.

\subsection{Results on $J / \psi$ production from $\mathbf{d}+$ Au collisions}

A large $J / \psi$ data set for $\mathrm{d}+\mathrm{Au}$ has been obtained for the first time in the 2008 run. PHENIX has released d+Au $R_{C P}$ data for $J / \psi$ production [17] in nine rapidity bins over $|y|<2.4$. Systematic uncertainties associated with the beam luminosity, detector acceptance, trigger efficiency, and tracking efficiency cancel when $R_{C P}$, the ratio of central to peripheral events, if formed. There is a remaining systematic uncertainty due to the centrality dependence of the tracking and particle identification efficiencies. However, there are significant systematic uncertainties in the centrality 
dependence of $R_{C P}$ due to the use of a Glauber model to calculate the average number of nucleonnucleon collisions as a means of estimating the relative normalization between different centrality bins. The systematic uncertainty due to the Glauber calculation is independent of rapidity.

The PHENIX d+Au $R_{C P}$ data have been independently fitted at each of the nine rapidities [19] employing a model including shadowing and $J / \psi$ absorption. The model calculations use the EKS98 and nDSg shadowing parameterizations with $0 \leq \sigma_{\mathrm{abs}} \leq 15 \mathrm{mb}$. The best fit absorption cross section was determined at each rapidity, along with the $\pm 1 \sigma$ uncertainties associated with a) rapidity-dependent systematic uncertainties and b) rapidity-independent systematic uncertainties. The results are shown in Fig. 4. The most notable feature is the stronger effective absorption cross section at forward rapidity, similar to the behavior observed at lower energies [10].

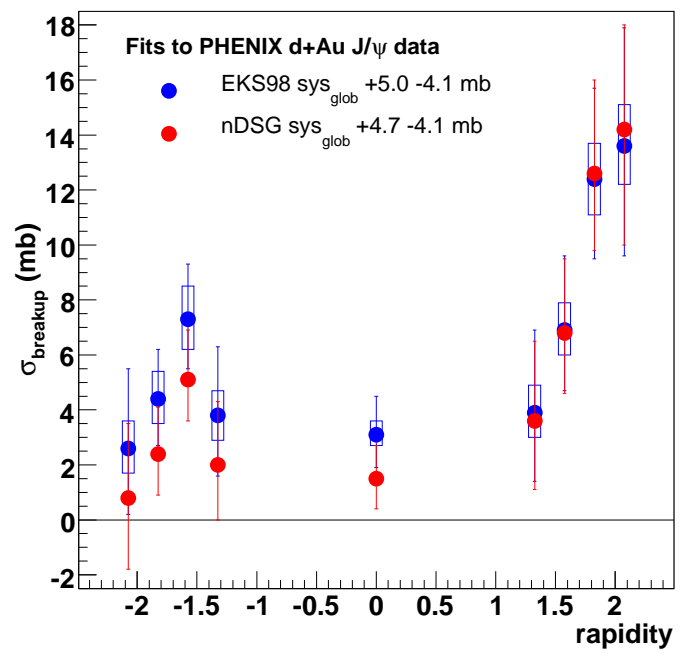

Figure 4: The effective absorption cross section as a function of rapidity extracted from PHENIX d+Au $R_{C P}$ data using the EKS98 and nDSg shadowing parameterizations. The vertical bars show uncorrelated point-to-point uncertainties, the boxes show correlated uncertainties, and the global uncertainties are given in the legend.

Note the large global systematic uncertainty in $\sigma_{\text {abs }}$ extracted from the PHENIX $R_{C P}$ data, dominated by the uncertainty in the Glauber estimate of the average number of collisions at each centrality. Although it does not affect the shape of the rapidity dependence of $\sigma_{\text {abs }}^{J / \psi}$, it results in considerable uncertainty in the magnitude of the effective absorption cross section.

\subsection{Results on $J / \psi$ production in Au+Au collisions}

PHENIX has published the centrality dependence of $R_{A A}$ for $\mathrm{Au}+\mathrm{Au}$ collisions using $\mathrm{Au}+\mathrm{Au}$ data from the 2004 RHIC run and $p p$ data from the 2005 run [20]. $R_{A A}$, the nuclear modification factor, is given by the ratio between the yield in A-A per $N-N$ collision, and the $p p$ yield. The results are shown in Fig.5. The suppression $\left(R_{A A}<1\right)$ is considerably stronger at forward rapidity than at midrapidity. The significance of this difference with respect to hot matter effects is not clear, however, unless the suppression due to cold nuclear matter effects is better known. 


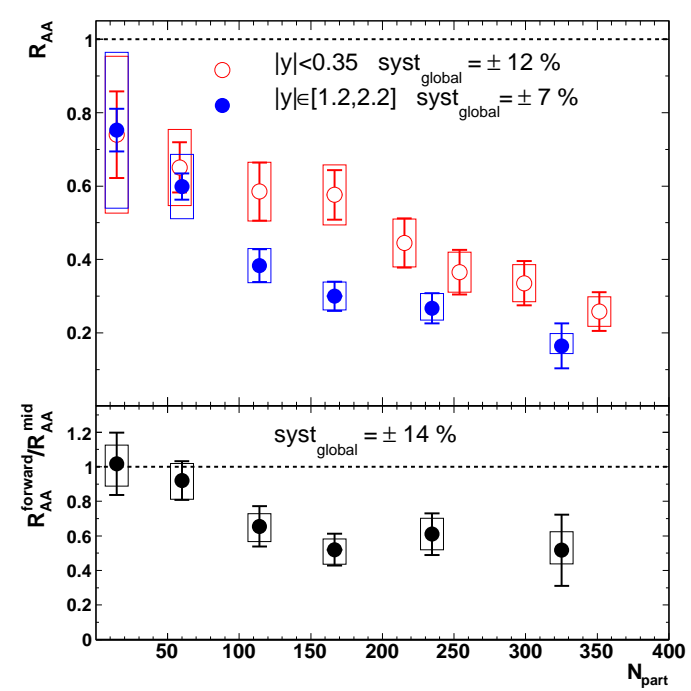

Figure 5: The PHENIX Au+Au $R_{A A}$ as a function of centrality for $|y|<0.35$ and $1.2<|y|<2.2$.

To estimate the cold nuclear matter contribution to the $\mathrm{Au}+\mathrm{Au} J / \psi R_{A A}$ the $\mathrm{d}+\mathrm{Au} J / \psi R_{C P}$ data were extracted using the EKS98 and nDSg shadowing parameterizations, as described earlier, exception that, in this case, the $\sigma_{\mathrm{abs}}^{J / \psi}$ values in $\mathrm{d}+\mathrm{Au}$ collisions were fitted independently in three rapidity intervals: $-2.2<y<-1.2 ;|y|<0.35$ and $1.2<y<2.2$. In effect, this tunes the calculations to reproduce the $\mathrm{d}+\mathrm{Au} R_{C P}$ independently in each of the three rapidity windows in which the $\mathrm{Au}+\mathrm{Au} R_{A A}$ data were measured. The cold nuclear matter $R_{A A}$ for $\mathrm{Au}+\mathrm{Au}$ collisions was then estimated in a Glauber calculation using the fitted absorption cross sections and the centralitydependent $R_{p \text { Au }}$ calculated using EKS98 and $\mathrm{nDSg}$ shadowing parameterizations. The uncertainty in the calculated CNM $R_{A A}$ was estimated by repeating the calculation with the best fit $\sigma_{\text {abs }}^{J / \psi}$ values varied within the rapidity-dependent systematic uncertainty determined when fitting the $\mathrm{d}+\mathrm{Au} R_{C P}$.

The $J / \psi$ suppression beyond CNM effects in $\mathrm{Au}+\mathrm{Au}$ collisions can be estimated by dividing the measured $R_{A A}$ by the estimates of the CNM $R_{A A}$. The result for EKS98 is shown in Fig. 6 . The result for $\mathrm{nDSg}$ is nearly identical.

Assuming that the final PHENIX $R_{\mathrm{dAu}}$ confirms the strong suppression at forward rapidity seen in $R_{C P}$, it would suggest that the stronger suppression seen at forward/backward rapidity in the PHENIX Au+Au $R_{A A}$ data is primarily due to cold nuclear matter effects. The suppression due to hot matter effects seems to be comparable at midrapidity and at forward/backward rapidity.

\section{Anomalous suppression: SPS vs RHIC}

The preliminary PHENIX d+Au results at $\sqrt{s}=200 \mathrm{GeV}$ are, for the first time, based on a high-statistics sample [19]. Comparing these results with the previous $\mathrm{Au}+\mathrm{Au}$ data gives an estimate of the magnitude of the anomalous $J / \psi$ suppression at RHIC. The newly available NA60 $p A$ results at $158 \mathrm{GeV}$, described in Section 2, allow significant comparison between the centrality dependence of the anomalous suppression at the SPS and at RHIC. Work is in progress to make such a comparison as a function of several variables of interest such as the charged particle multiplicity, $d N_{\mathrm{ch}} / d \eta$, and the Bjorken energy density reached in the collision. The anomalous suppression 


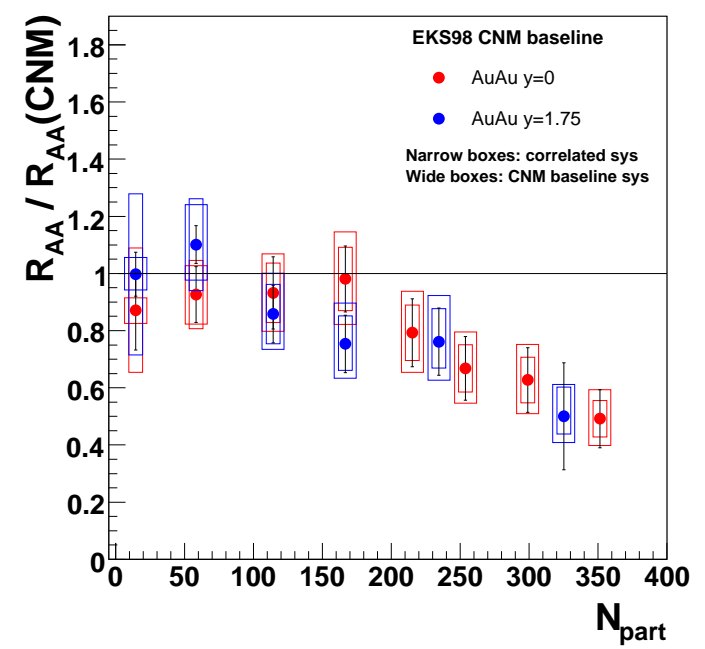

Figure 6: The estimated $\mathrm{Au}+\mathrm{Au}$ suppression relative to the cold nuclear matter $R_{A A}$ as a function of centrality for $|y|<0.35$ and $1.2<|y|<2.2$. The systematic uncertainty of the baseline cold nuclear matter $R_{A A}$ is depicted by the wide box around each point. The narrow box is the systematic uncertainty in the $\mathrm{Au}+\mathrm{Au}$ $R_{A A}$.

patterns in $\mathrm{In}+\mathrm{In}$ and $\mathrm{Pb}+\mathrm{Pb}$ collisions at the SPS and the midrapidity $\mathrm{Au}+\mathrm{Au}$ results at RHIC are presented as a function of $d N_{\mathrm{ch}} / d \eta$ in Fig. 7 [21]. Note that the magnitude of the anomalous $J / \psi$ suppression is practically system and $\sqrt{s}$-independent when expressed as a function of $d N_{\mathrm{ch}} /\left.d \eta\right|_{\eta=0}$.

\section{Heavy quarkonium in nuclear collisions at LHC energy}

With the advent of the LHC, it is expected to create a strongly interacting medium with an initial temperature well beyond the critical value for deconfinement $\left(T_{\mathrm{c}} \sim 170 \mathrm{MeV}\right)$. Thanks to the high production cross sections, it will be possible to measure with good statistics, for the first time, the various bottomonium states. In particular, the tightly bound $\Upsilon(1 S)$ should melt only at temperatures above $2 T_{\mathrm{c}}[22]$ and represents therefore an ideal "thermometer" for $\mathrm{Pb}-\mathrm{Pb}$ collisions at the LHC, which will be first studied at $\sqrt{s}=2.75 \mathrm{TeV} /$ nucleon at the end of 2010, and later on up to $\sqrt{s}=5.5 \mathrm{TeV} /$ nucleon. The $c$-quark multiplicity is expected to be very high at LHC energy, of the order of 100 pairs for central $\mathrm{Pb}-\mathrm{Pb}$ collisions. Therefore, when the system hadronizes, $c \bar{c}$ pairs may coalesce and lead to an increase with centrality of the charmonium yield [23]. This effect may be so strong to mask the dissociation due to color screening in the Quark-Gluon Plasma phase.

Both CMS [24] and ATLAS [25] plan to carry out an accurate measurement of the heavy quarkonium states in nuclear collisions, at central rapidities. ALICE [26], the dedicated heavyion experiment at the LHC, will measure charmonia and bottomonia at both central and forward rapidities. The expected physics performance are shortly described in the following section.

\subsection{Charmonium and bottomonium production in ALICE}

Heavy quarkonia will be measured in ALICE in the central barrel, in the pseudorapidity range 


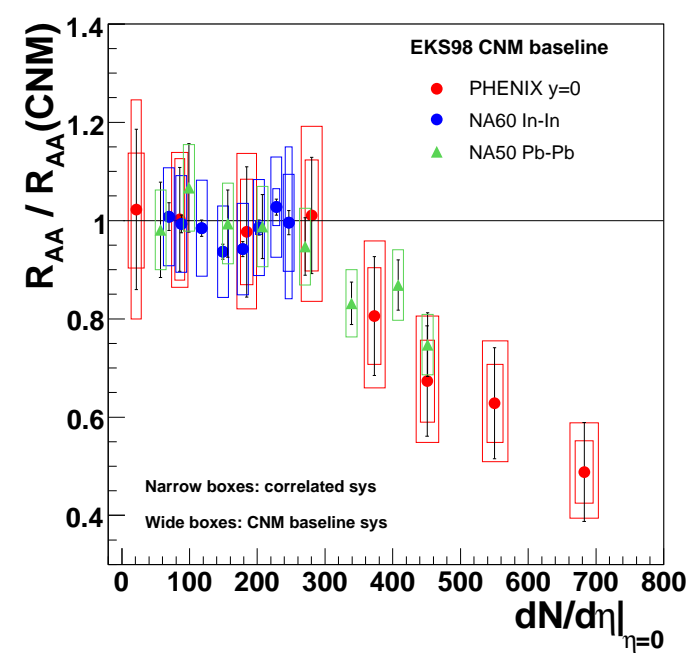

Figure 7: Comparison of the anomalous suppression at the SPS and RHIC as a function of $d N_{\mathrm{ch}} / d \eta$ at $\eta=0$.

$-0.9<\eta<0.9$, and in the forward muon arm, with a $2.5<\eta<4$ coverage. In the central barrel, heavy quarkonia will be detected through the $e^{+} e^{-}$decay. ALICE can push its $p_{\mathrm{T}}$ reach for charmonium down to $p_{\mathrm{T}} \sim 0$. Electron identification is performed jointly in the TPC through the $d E / d x$ measurement and in the Transition Radiation Detector (TRD). In the forward region, quarkonia will be studied via their decay into muon pairs. Muons with momenta larger than $4 \mathrm{GeV} / \mathrm{c}$ are detected by means of a spectrometer which includes a $3 \mathrm{Tm}$ dipole magnet, a front absorber, a muon filter, tracking (Cathode Pad Chambers, CPC) and triggering (Resistive Plate Chambers, RPC) devices.

At the nominal luminosity $\left(L=5 \cdot 10^{26} \mathrm{~cm}^{-2} \mathrm{~s}^{-1}\right)$, the expected $\mathrm{J} / \psi$ statistics in $\mathrm{Pb}+\mathrm{Pb}$ for a $10^{6}$ $\mathrm{s}$ data taking, corresponding to the yearly running time with the $\mathrm{Pb}$ beam, is of the order of $7 \cdot 10^{5}$ events in the forward muon arm. The mass resolution will be $\sim 70 \mathrm{MeV} / \mathrm{c}^{2}$ [27]. A simulation of the various background sources to the muon pair invariant mass spectrum in the $\mathrm{J} / \psi$ region (including combinatorial $\pi$ and $K$ decays, as well as semileptonic decays of open heavy flavours) shows that the ratio signal/background (S/B) ranges from 0.13 to $\sim 7$ when moving from central to peripheral collisions. With such statistics and S/B values, it will be possible to study the proposed theoretical scenarios for the modification of the $\mathrm{J} / \psi$ yield in the hot medium. The transverse momentum distributions can be addressed with reasonable statistics even for the relatively less populated peripheral $\mathrm{Pb}+\mathrm{Pb}$ collisions. In particular, for collisions with an impact parameter $b>12 \mathrm{fm}$, it is expected to have more than 1000 events with $p_{\mathrm{T}}>8 \mathrm{GeV} / \mathrm{c}$. Finally, a study of the $\mathrm{J} / \psi$ polarization will be performed by measuring the angular distribution of the decay products. With the expected statistics, the polarization parameter $\lambda$ extracted from the fit $d \sigma / d \cos \theta=\sigma_{0}\left(1+\lambda \cos ^{2} \theta\right)$ can be measured, defining five impact parameter bins, with a statistical error $<0.05$ for each bin.

At central rapidity, the expected $\mathrm{J} / \psi$ statistics, measured for $10^{6} \mathrm{~s}$ running time at the nominal luminosity, is about $2 \cdot 10^{5}$ for $10^{8}$ triggered $10 \%$ most central events. The mass resolution will be $\sim 30 \mathrm{MeV} / \mathrm{c}^{2}$ [28]. The background under the $\mathrm{J} / \psi$ peak is dominated by misidentified pions, but is anyway at a rather comfortable level $(\mathrm{S} / \mathrm{B}=1.2)$. As for the forward region, it will therefore be 
possible to test the proposed theoretical models. The $\mathrm{S} / \mathrm{B}$ ratio is expected to increase as a function of $p_{\mathrm{T}}$, reaching a value of $\sim 5$ at $10 \mathrm{GeV} / \mathrm{c}$. The expected statistics at that $p_{\mathrm{T}}$ is still a few hundred counts, implying that differential $\mathrm{J} / \psi$ spectra can also be studied.

Concerning bottomonium production, with the foreseen muon pair invariant mass resolution $\left(\sim 100 \mathrm{MeV} / \mathrm{c}^{2}\right)$ the $\Upsilon$ states can be clearly separated. The expected yields are of the order of $7 \cdot 10^{3}$ events for the $\Upsilon(1 \mathrm{~S})$ in $\mathrm{Pb}+\mathrm{Pb}$ collisions, and of a factor $\sim 4$ and $\sim 6.5$ smaller for the higher mass resonances $\Upsilon(2 S)$ and $\Upsilon(3 S)$, respectively [29]. The S/B ratios will be more favorable than for the $\mathrm{J} / \psi(\sim 1.7$ for the $\Upsilon(1 \mathrm{~S})$ in central collisions).

At midrapidity, a possibility of measuring the $\Upsilon$ states is tightly related to the foreseen implementation of a Level-1 trigger on electrons [28]. In a $\mathrm{Pb}+\mathrm{Pb}$ run a significant $\Upsilon(1 \mathrm{~S})$ sample (several thousand events) can be collected, with a comfortable $\mathrm{S} / \mathrm{B} \sim 1$. The statistics for the higher mass resonances depends crucially on the production mechanism. Assuming, as for $\Upsilon(1 \mathrm{~S})$, binary scaling, a measurement of $\Upsilon(2 S)$ looks very promising $(\sim 1000$ events with $\mathrm{S} / \mathrm{B}=0.35)$.

Finally, the study of $p p$ collisions is essential in order to provide reference data for the interpretation of nuclear collision results (also $p A$ collisions are foreseen, but not in the first years of operation of the LHC). At $\sqrt{s}=14 \mathrm{TeV}$, the nominal LHC energy, a typical data taking period of one year at $L=3 \cdot 10^{30} \mathrm{~cm}^{-2} \mathrm{~s}^{-1}$ (the maximum luminosity the ALICE experiment can stand) gives an integrated luminosity of $30 \mathrm{pb}^{-1}$, corresponding to $\sim 2.8 \cdot 10^{6}$ events [30] in the muon arm. The corresponding dimuon invariant mass spectrum, for opposite-sign pairs, is shown in Fig. 8.

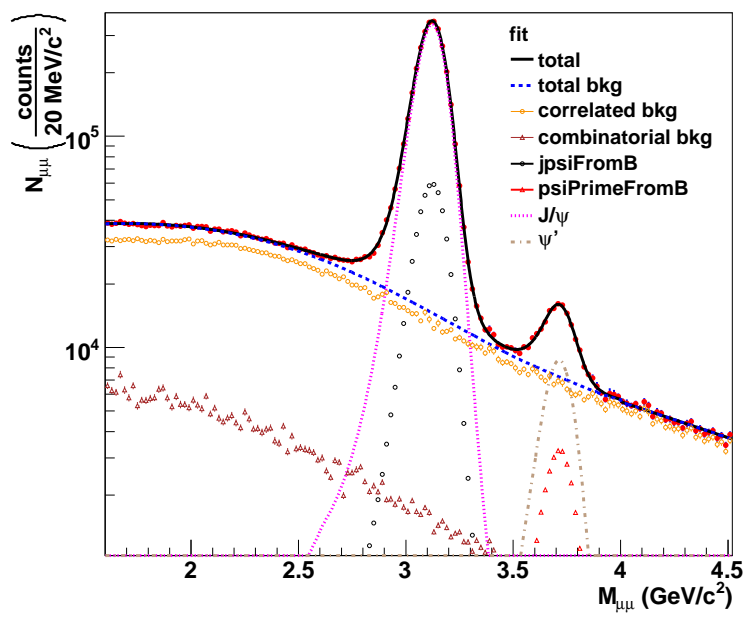

Figure 8: Opposite sign dimuon mass spectrum in p-p collisions at $\sqrt{s}=14 \mathrm{TeV}$ for a $10^{7} \mathrm{~s}$ running time at $L=3 \cdot 10^{30} \mathrm{~cm}^{-2} \mathrm{~s}^{-1}$.

In the central arm, a sample of a few $1000 \mathrm{~J} / \psi$ events is expected to be acquired in minimum bias p-p collisions. With such statistics it will be possible to measure $d \sigma / d y$ at midrapidity. With the implementation of a Level-1 trigger on electrons, and assuming a conservative trigger efficiency of $10 \%$, one would get about $7 \cdot 10^{5} \mathrm{~J} / \psi$.

\section{Conclusions}

A systematic study of $\mathrm{J} / \psi$ suppression in nuclear collisions has been carried out at SPS and 
RHIC energies. The observed suppression is not compatible with an effect due only to charmonium interaction in cold nuclear matter. When expressed as a function of the charged multiplicity at midrapidity, the $\mathrm{J} / \psi$ suppression is practically $\sqrt{s}$-independent. These findings will be further tested at LHC energies, where $\mathrm{Pb}+\mathrm{Pb}$ collisions at $\sqrt{s}=2.75 \mathrm{TeV} /$ nucleon will be studied, for the first time, at the end of 2010. Furthermore, at the LHC, bottomonium states will be copiously produced, and their anomalous suppression, expected to set in, for the $\Upsilon(1 S)$ state, at temperatures much higher than $T_{\mathrm{c}}$, should be observed.

\section{References}

[1] N. Brambilla et al. [Quarkonium Working Group], Heavy quarkonium physics, arXiv: hep-ph/0412158.

[2] T. Matsui and H. Satz, J/ $\psi$ suppression by quark-gluon plasma formation, Phys. Lett. B 178 (1986) 416.

[3] E. Scomparin [NA60 Collaboration], J/ $\psi$ production in $p$-A collisions at 158 and $400 \mathrm{GeV}$ : recent results from the NA60 experiment, Nucl. Phys. A 830 (2009) 239c [arXiv: 0907.3682 [nucl-ex] ].

[4] R. Arnaldi et al. [NA60 Collaboration], J/ $\psi$ production in indium-indium collisions at 158 GeV/nucleon, Phys. Rev. Lett. 99 (2007) 132302.

[5] R. Arnaldi [NA60 Collaboration], $J / \psi$ production in $p-A$ and A-A collisions at fixed target experiments, Nucl. Phys. A 830 (2009) 345c [arXiv:0907.5004 [nucl-ex] ].

[6] B. Alessandro et al. [NA50 Collaboration], A new measurement of $\mathrm{J} / \psi$ suppression in $\mathrm{Pb}-\mathrm{Pb}$ collisions at $158 \mathrm{GeV}$ per nucleon, Eur. Phys. J. C 39, 335 (2005) [arXiv: hep-ex/0412036] .

[7] B. Alessandro et al. [NA50 Collaboration], Charmonium production and nuclear absorption in p-A interactions at $450 \mathrm{GeV}$, Eur. Phys. J. C 33 (2004) 31.

[8] B. Alessandro et al. [NA50 Collaboration], $J / \psi$ and $\psi$ ' production and their normal nuclear absorption in proton-nucleus collisions at $400 \mathrm{GeV}$, Eur. Phys. J. C 48 (2006) 329 [arXiv:nucl-ex/0612012].

[9] B. Alessandro et al. [NA50 Collaboration], Charmonia and Drell-Yan production in proton-nucleus collisions at the CERN SPS, Phys. Lett. B 553, 167 (2003).

[10] M. J. Leitch et al. [FNAL E866/NuSea collaboration], Measurement of $J / \psi$ and $\psi$ ' suppression in $p-A$ collisions at $800 \mathrm{GeV} / c$, Phys. Rev. Lett. 84 (2000) 3256 [arXiv:nucl-ex/9909007] .

[11] J. Badier et al. [NA3 Collaboration], Experimental J/ $\psi$ hadronic production from $150 \mathrm{Gev} / \mathrm{c}$ to 280 Gev/c, Z. Phys. C 20 (1983) 101.

[12] I. Abt et al. [HERA-B Collaboration], Kinematic distributions and nuclear effects of J/ $\psi$ production in $920 \mathrm{GeV}$ fixed-target proton-nucleus collisions, Eur. Phys. J. C 60 (2009) 525 [arXiv:0812.0734 [hep-ex]].

[13] R. Vogt, The $x_{\mathrm{F}}$ dependence of $\psi$ and Drell-Yan production, Phys. Rev. C 61 (2000) 035203 [arXiv: hep-ph/9907317].

[14] K. J. Eskola, V. J. Kolhinen and C. A. Salgado, The scale dependent nuclear effects in parton distributions for practical applications, Eur. Phys. J. C 9 (1999) 61 [arXiv:hep-ph/9807297] . 
[15] K. J. Eskola, H. Paukkunen and C. A. Salgado, An improved global analysis of nuclear parton distribution functions including RHIC data, JHEP 0807 (2008) 102 [ arXiv: 0802.0139 [hep-ph] ].

[16] R. Arnaldi, P. Cortese and E. Scomparin, The role of parton shadowing in the comparison of $p$-A and A-A results on J/ $\psi$ suppression at SPS energy, Phys. Rev. C 81 (2010) 014903 [arXiv:0909.2199 [hep-ph]].

[17] C. L. da Silva [PHENIX Collaboration], Quarkonia measurement in $p+p$ and $d+A u$ collisions at $\sqrt{s}=200 \mathrm{GeV}$ by PHENIX detector, Nucl. Phys. A 830 (2009) 227c [arXiv: 0907.4696 [nucl-ex] ].

[18] C. Perkins [STAR Collaboration], J/ $\psi$ production in $p+p$ and $d+A u$ collisions at $\sqrt{s_{N N}}=200$ GeV at STAR, Nucl. Phys. A 830 (2009) 231c [arXiv:0907.4396 [nucl-ex] ].

[19] A. D. Frawley, talk at ECT* workshop on Quarkonium Production in Heavy-Ion Collisions, Trento (Italy), May 25-29, 2009 and at Joint CATHIE-INT mini-program “Quarkonia in Hot QCD”, June 16-26, 2009

[20] A. Adare et al. [PHENIX Collaboration], J/ $\psi$ production vs centrality, transverse momentum, and rapidity in $A u+A u$ collisions at $\sqrt{s_{N N}}=200 \mathrm{GeV}$, Phys. Rev. Lett. 98, 232301 (2007) [arXiv:nucl-ex/0611020].

[21] R. Arnaldi [NA60 Collaboration], talk at ECT* workshop on Quarkonium Production in Heavy-Ion Collisions, Trento (Italy), May 25-29, 2009.

[22] Á. Mócsy and P. Petreczky, Color screening melts quarkonium, Phys. Rev. Lett. 99 (2007) 211602 [arXiv:0706.2183 [hep-ph]].

[23] A. Andronic, P. Braun-Munzinger, K. Redlich and J. Stachel, Statistical hadronization of heavy quarks in ultra-relativistic nucleus-nucleus collisions, Nucl. Phys. A 789 (2007) 334 [arXiv:nucl-th/0611023].

[24] O. Kodolova et al. [CMS Collaboration], Heavy ion physics with CMS, Nucl. Phys. A 830 (2009) 97c [arXiv:0907.4492 [nucl-ex]].

[25] J. Dolejsí et al. [ATLAS Collaboration], Status of ATLAS and preparation for the Pb-Pb run, Nucl. Phys. A 830 (2009) 89c [arXiv:0907.4597 [nucl-ex] ] .

[26] P.G. Kuijer et al. [ALICE Collaboration], Commissioning and prospects for early physics with ALICE, Nucl. Phys. A 830 (2009) 81c [arXiv:0907.5060 [nucl-ex] ] .

[27] F. Carminati et al. [ALICE Collaboration], ALICE Physics Performance Report, Vol. I, J. Phys. G 30 (2004) 1517.

B. Alessandro et al. [ALICE Collaboration], ALICE Physics Performance Report, Vol. II, J. Phys. G 32 (2006) 1295.

[28] W. Sommer et al., Quarkonia measurements with the central detectors of ALICE, arXiv:nucl-ex/0702045.

[29] S. Grigoryan and A. De Falco, Capabilities of the ALICE Muon Spectrometer to measure quarkonia producttion in Pb-Pb collisions at 5.5 TeV, ALICE-INT-2008-016.

[30] D. Stocco et al., Quarkonia detection with the ALICE muon spectrometer in pp collisions at $\sqrt{s}=14$ $\mathrm{TeV}$, ALICE-INT-2006-029. 